University of Wollongong

Research Online

Faculty of Engineering and Information

Faculty of Engineering and Information

Sciences - Papers: Part B

Sciences

2018

Using Vibration Motors to Create Tactile Apparent Movement for

Transradial Prosthetic Sensory Feedback

Benjamin Stephens-Fripp

University of Wollongong, bsf147@uowmail.edu.au

Rahim Mutlu

University of Wollongong, rmutlu@uow.edu.au

Gursel Alici

University of Wollongong, gursel@uow.edu.au

Follow this and additional works at: https://ro.uow.edu.au/eispapers1

Part of the Engineering Commons, and the Science and Technology Studies Commons

Research Online is the open access institutional repository for the University of Wollongong. For further information contact the UOW Library: research-pubs@uow.edu.au 


\title{
Using Vibration Motors to Create Tactile Apparent Movement for Transradial Prosthetic Sensory Feedback
}

\author{
Abstract \\ It has been reported in the literature that sensory information is a valuable and desired form of feedback \\ for prosthetic users. Communication of how the arm moves can reduce cognitive load, reduce the need \\ for visual attention and help the user predict the initial grasping force. In this paper, a new method of \\ communicating movement sensations is presented through the application of tactile apparent \\ movement. By overlapping vibration created by arrays of linear resonant actuators, a stroking movement \\ can be felt on the user's arm. The results show potential for a low cost and light weight system that can \\ communicate stimulations for up to three degrees of actuation in a prosthetic.

\section{Disciplines} \\ Engineering | Science and Technology Studies

\section{Publication Details} \\ Stephens-Fripp, B., Mutlu, R. \& Alici, G. (2018). Using Vibration Motors to Create Tactile Apparent \\ Movement for Transradial Prosthetic Sensory Feedback. IEEE International Conference on Biomedical \\ Robotics and Biomechatronics (Biorob) (pp. 213-218). United States: IEEE.
}




\title{
Using Vibration Motors to Create Tactile Apparent Movement for Transradial Prosthetic Sensory Feedback
}

\author{
Benjamin Stephens-Fripp, Rahim Mutlu, and Gursel Alici
}

\begin{abstract}
It has been reported in the literature that sensory information is a valuable and desired form of feedback for prosthetic users. Communication of how the arm moves can reduce cognitive load, reduce the need for visual attention and help the user predict the initial grasping force. In this paper, a new method of communicating movement sensations is presented through the application of tactile apparent movement. By overlapping vibration created by arrays of linear resonant actuators, a stroking movement can be felt on the user's arm. The results show potential for a low cost and light weight system that can communicate stimulations for up to three degrees of actuation in a prosthetic.
\end{abstract}

\section{INTRODUCTION}

Current prosthesis rely on visual information to provide feedback for the user. However, visual feedback alone does not provide enough information to control and manipulate prosthetic devices [1] and prosthetic users have a desire to reduce the reliance on visual feedback [2]. The lack of sensory feedback contributes towards the high rejection rates of prosthetic hands [3, 4], as well as decreasing the user's sense of embodiment [5-7].

Prosthetic users have identified the grasping force and position feedback as the two highest priorities [8]. The majority of literature on communicating proprioceptive information sends position information back to the user [9]. Velocity feedback, however, can also be used to help the user understand the change in position [10]. In addition, the initial grasping force can be predicted from proprioceptive information [11], e.g. by providing users on the velocity of movement of prosthetic digits [12]. Ninu et al. [12] hypothesise that it is highly possible to predict initial grasping force based on information immediately preceding the grasp, i.e. the velocity of our fingers prior to making contact with the object. They also noted from their results that velocity feedback was the most important feature to help control initial grasping force, however, this was with a 1-DOF hand.

There are a number of tactile sensing methods found within the literature [9]. Multiple studies examine the use of vibrational feedback, as small and lightweight commercially available vibration motors can be easily be applied to the skin, and the vibration sensation is preferred over electrotactile

B. Stephens-Fripp, G. Alici and R. Mutlu are with the Intelligent Nano-Tera Systems Research Laboratory, based at the School of Mechanical, Materials, Mechatronic and Biomedical Engineering, University of Wollongong, Australia; and are part of the ARC Centre of Excellence for Electromaterials Science, University of Wollongong, Australia. (Corresponding author: Gursel Alici - gursel@uow.edu.au). stimulation [13]. The majority of the literature, however, focuses on the use of vibration to communicate grasping force [12, 14-19], but some have studied its application in feeding back proprioceptive information $[10,12,20]$ as well as modality matched texture feedback [21, 22]. Recently, Marasco et al. [23] demonstrated the ability to induce kinaesthesia in patients by vibrating their tendons, however, this required patients to undergo targeted reinnervation surgery. Skin stretch has also been applied to communicate proprioceptive information, but most studies only communicate one sensation at a time [24, 25], as suitable for a prosthetic device with one degree of actuation. Akhtar et al. [26] used a passive mechanical skin stretch for proprioception which demonstrated positive results for position recognition. However, they rely on pads to be adhered to the skin with adhesive, which may be impractical for repeated daily use and may result in skin irritation [27].

Tactile apparent movement occurs when two discrete stimulations occur sequentially on the skin and are perceived to be a stroking sensation [28]. Chen et al. [29] uses tactile apparent movement with four vibration tactors to communicate perception of movement around an ankle for correction walking movements. Each stimulation has a period of overlapping with the previous stimulation to create this movement sensation. The Interstimulus Onset Interval (ISOI) is the time required of overlapping stimulations to achieve perception of movement, as shown in Figure 1.

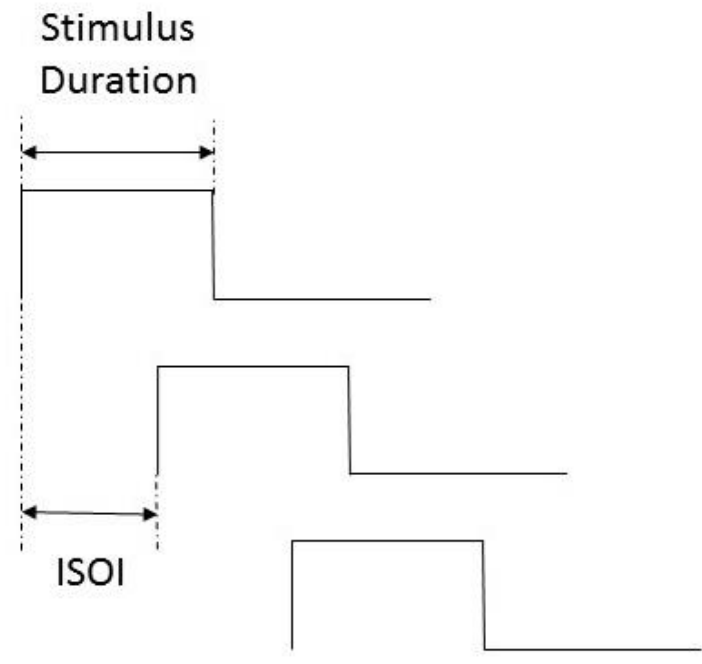

Figure 1 - Three vibration pulses to create Tactile Apparent Movement. (Adapted from [29]) 
If the ISOI is too short it will be perceived as simultaneous stimulation, if the ISOI is too long it will be perceived as successive stimulation [30]. Increasing the number of stimulators increases the accuracy of identify apparent tactile motion [31] and results in a shorter ISOI required. In a pilot study, Kirman reported that accuracy in detecting apparent motion is not dependent upon the spacing of the stimulators [29] between 0.2 " $(5.08 \mathrm{~mm})$ and 2 "(50.8mm).

In this study, we propose a new application of tactile apparent movement, by using it to communicate the movement of individual prosthetic digits.

Drawbacks of vibration motors include the delay in response time and the perceived frequency being dependent upon how tight the vibration motor is pressed against the skin [32]. However, since the perceived location vibrations will be used within this study to communicate movement, the perceived frequency is not important and the issue of the response time is of a lower priority. However, to increase response time, the Linear Resonant Actuators (LRAs) will be driven by haptic drivers, which uses overdrive voltage and braking to minimise start up and stopping times.

\section{METHOD}

The proposed vibration array system is shown in Figure 2. It consists of 9, 8mm LRA vibration motors (Jinglong Machinery and Electronics Inc) arranged in three rows of three motors spaced $22 \mathrm{~mm}$ apart (centre to centre). The original setup consisted of an array of four motors spaced $15 \mathrm{~mm}$ apart, however, initial testing demonstrated that this close spacing was difficult to identify movement directions. To increase response times, each motor is controlled by a Haptic Driver (Texas Instruments DRV2605L) through an I2C interface. The haptic driver also enables the use of auto resonant frequency tracking through the back EMF generated the motors, to ensure the LRA motors are operating at their optimum frequency. However, since each haptic driver has the same I2C address, they communicate to the NI MyRio microcontroller through three daisy chained I2C Multiplexers. This communication hierarchy is shown in Figure 4. A surfboard leash cuff (Smart Leash Co.) was used to hold them firmly against the user's skin, as shown in Figure 3. A 3D printed 5mm thick backing plate made of Ninjaflex with solid infill was placed between the motors and the cuff to reduce its soft cushioning from absorbing the vibrations. When attached to the arm, the three vibration arrays ran longitudinal to the arm, as shown in Figure $3 b$.

The setup was chosen to represent information from a prosthetic hand with three Degrees Of Actuation (3DOA); where the three motors independently moving the thumb, pointer and then the remaining three fingers together; and each tactor array corresponds to the movement of one of these motors. Recognition of six different grip patterns, shown in Figure 5, was tested: thumb only, pointer only, pistol grip (closing remaining three fingers only), fine grip (closing thumb and pointer), tool grip (closing thumb and remaining three fingers) and power grip (closing all fingers). These grip patterns were chosen due to being previous employed in other sensory feedback literature [26, 33]. Each of these grips were tested in an opening movement, represented by the vibration travelling up the arm towards the shoulder, and in a closing movement represented by the vibration travelling down the arm towards the hand. As a result of initial testing, the stimulus duration was set at $200 \mathrm{~ms}$ with an ISOI of $180 \mathrm{~ms}$.

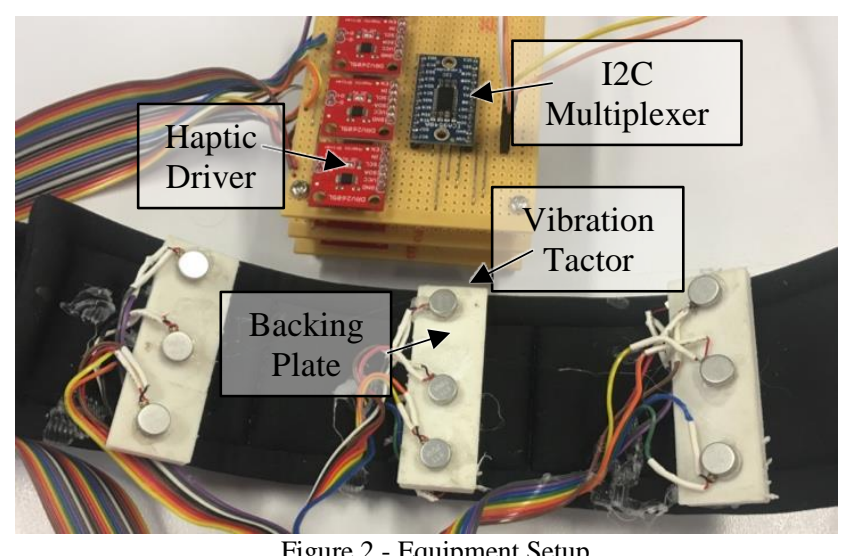

Figure 2 - Equipment Setup
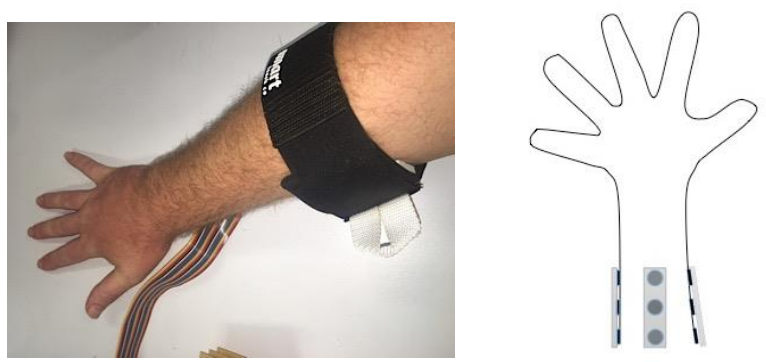

(a)

(b)

Figure 3 - Vibrational Arrays

(a) Placement on Arm and, (b) Tactor Location (Underside of Arm)

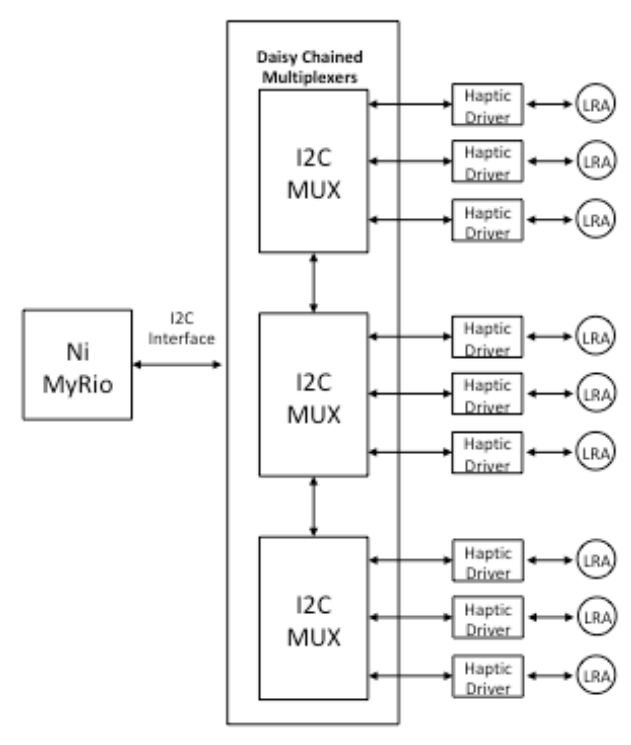

Figure 4 - Communication Heirarchy Setup 
In the training phase, each of the different six movements were demonstrated to the user in both an upwards and a downwards direction. Prior to the stimulation, the user was told verbally which grip and direction would be sent.

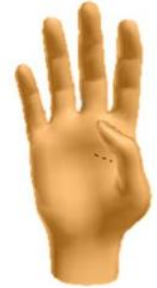

(a)

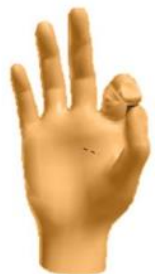

(d)

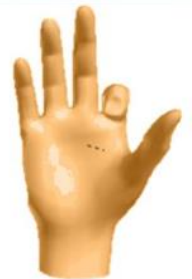

(b)

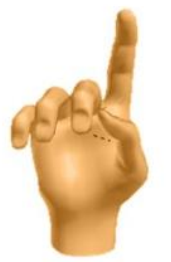

(e)

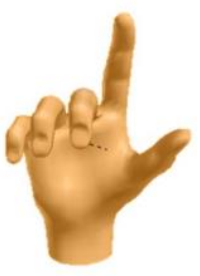

(c)

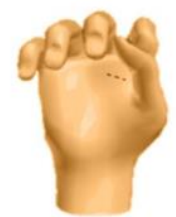

(f)
Figure 5 - Hand Grips: (a) Thumb Only, (b) Pointer only, (c) Pistol Grip, (d) Fine Grip, (e) Tool Grip and, (f) Power Grip

Each subject received eighteen different stimulations, consisting of the six different grip movements repeated three times, all presented in a randomised order. Half of the stimulations were randomly assigned an upward apparent movement direction, and half of the stimulations were randomly assigned a downwards apparent movement direction. Each subject received a different order and combination of movements. Each stimulation was communicated twice and a rest period of at least five-seconds occurred between movements for the subject to communicate the perceived grip movement and direction and to reduce desensitisation. A total of 10 subjects were tested, consisting of 8 males and 2 females, with no physical or cognitive impairment. Written informed consent was obtained from all individuals participating in the study and ethical approval was obtained from the University of Wollongong Human Research Ethics Committee.

\section{RESULTS AND DISCUSSION}

The average recognition rates for the vibration movements is shown in Table I and Figure 6. This is broken down into the performance of recognising the grip pattern sent only (not taking perceived direction into account), the direction of apparent movement only (ignoring which grip pattern was perceived), and performance of recognising the grip pattern and direction together correctly. A confusion matrix for grip recognition and the direction is shown in Figures 7 and 8, respectively. Although there was a very small training time, with each movement only receiving one demonstration, the system demonstrated its potential in the recognition rate. A longer training period, with either a virtual reality or myoelectric prosthetic arm, could help improve the recognition rate. Undergoing the testing process with feedback after each movement may also aid in the learning. The subjects were also only given two individual stimulations, however, the perception may change when using continual feedback.

TABLE I. RECOGNITION RATE OF VIBRATION MOVEMENTS

\begin{tabular}{|c|c|c|c|}
\hline Orientation & $\begin{array}{c}\text { Average \% } \\
\text { Recognition } \pm \\
\text { SD }\end{array}$ & $\begin{array}{c}\text { Median \% } \\
\text { Recognition }\end{array}$ & IQR \\
\hline Grip Only & $84 \% \pm 13 \%$ & $86 \%$ & $15 \%$ \\
\hline $\begin{array}{c}\text { Direction } \\
\text { only }\end{array}$ & $86 \% \pm 7 \%$ & $83 \%$ & $10 \%$ \\
\hline $\begin{array}{c}\text { Grip and } \\
\text { Direction }\end{array}$ & $72 \% \pm 11 \%$ & $78 \%$ & $19 \%$ \\
\hline
\end{tabular}

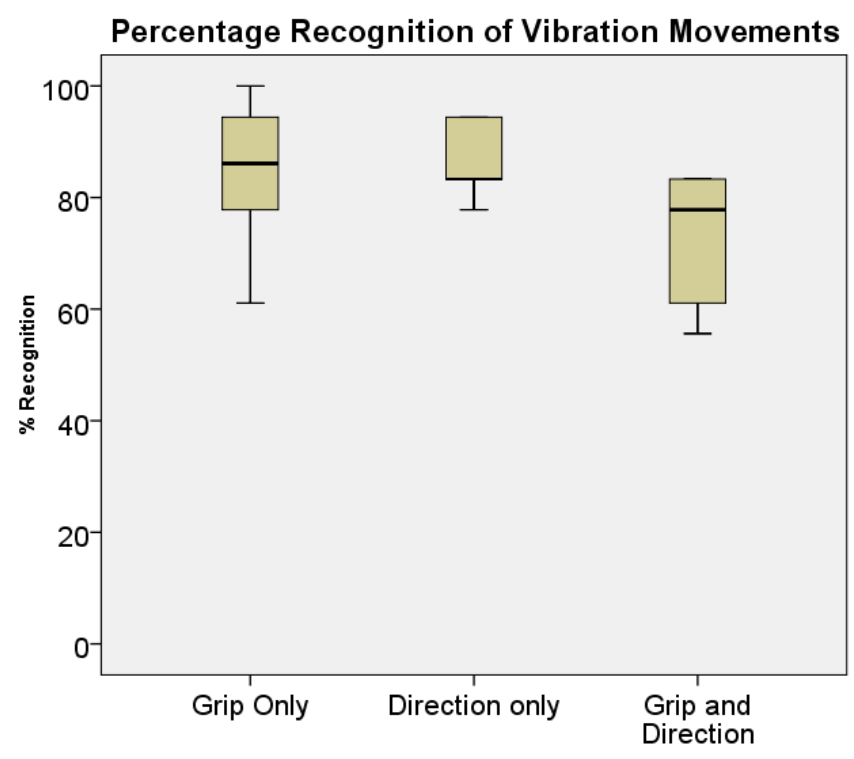

Figure 6 - Box Plot: Recognition Rate of Grip only; where: the dark line represents the median, The box indicates the Interquartile Range (IQR) and the whiskers represents either the $\mathrm{max} / \mathrm{min}$ or 1.5 times the IQR (whichever is closer)

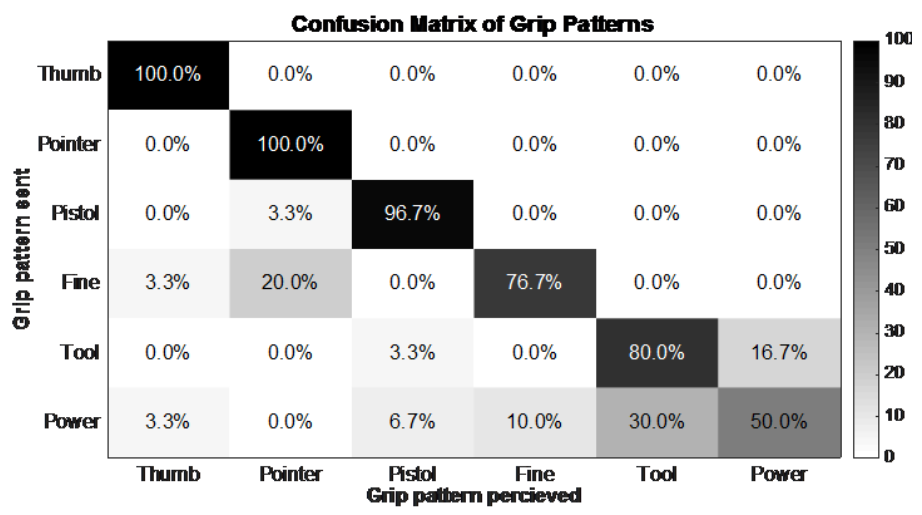

Figure 7 - Confusion Matrix of Grip Pattern Recognition 


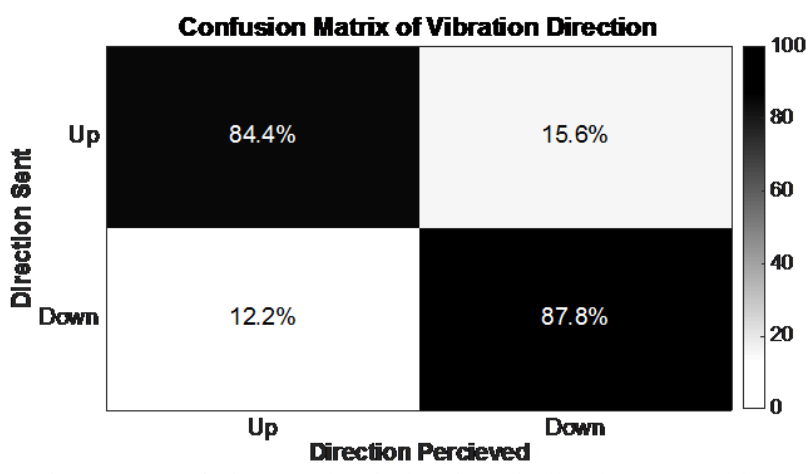

Figure 8 - Confusion Matrix of Direction of Vibration Recognition

TABLE II. RECOGNITION RATE OF EACH GRIP PATtERNS

\begin{tabular}{|c|c|c|c|}
\hline Grip pattern sent & \multicolumn{3}{|c|}{ Average \% Recognition } \\
\hline & $\begin{array}{c}\text { Grip } \\
\text { only }\end{array}$ & $\begin{array}{c}\text { Direction } \\
\text { only }\end{array}$ & $\begin{array}{c}\text { Grip and } \\
\text { Direction }\end{array}$ \\
\hline Thumb & $100 \%$ & $83 \%$ & $83 \%$ \\
\hline Pointer & $100 \%$ & $93 \%$ & $93 \%$ \\
\hline Pistol & $97 \%$ & $80 \%$ & $77 \%$ \\
\hline Fine & $77 \%$ & $93 \%$ & $73 \%$ \\
\hline Tool & $80 \%$ & $77 \%$ & $60 \%$ \\
\hline Power & $50 \%$ & $90 \%$ & $47 \%$ \\
\hline
\end{tabular}

As shown in Figure 6, the accuracy of the apparent direction is high with a small variance, however, a large variance is present in recognition of grips. As demonstrated in Figure 7 and Table II, a large amount of error in grip recognition occurred when interpreting the power grip stimulation. To reduce the error rate in grip recognition, more powerful vibration motors could be used. The perceived intensity of the vibration tactors is also dependent upon how hard they are pressed against the skin and their location on the arm. As a result, each row of tactors may be perceived as a different intensity which could contribute towards confusion when multiple rows are being stimulated simultaneously. To improve upon this, the intensity may require calibration to ensure the perceived level of stimulation is consistent between the different arrays. In addition, further experimentation is required to be conducted on the optimum locations on the arm for the vibration arrays. In this study, we used a standard armband for every subject, but this did not take into account the different arm sizes of the test subjects. Also different areas of the arm, (such as muscle, bone etc.) may have different perceptions of the vibration and this should be taken into consideration in future work.

The average recognition rate of grip and direction is low compared to grip only and direction only, suggesting that the errors in grip does not necessarily occur at the same time as errors in direction recognition. However, it is also worth noting that the recognition rate date for grip and direction is skewed right, as shown in Figure 6. As a result, the median may be a better indicator of performance than mean, which is closer to the other performance measures, as shown in Table I.

Additional experimentation is required on the combination of vibration duration and the corresponding ISOI. These results only examined movement at one speed. However, movement feedback will require a variety of speeds matching the movement of the prosthetic digits. In addition, the optimum ISOI may be different for each subject and may require personalised calibration.

Further work is required to optimise and improve this recognition results. Future research will focus at analysing the optimum number and spacing of vibration motors, separation of the backing for the vibration motor to minimise transfer of vibration, and seeking the best performing LRA with maximum response time. In addition, testing is required on amputees to ensure the same recognition rate, and incorporation into a feedback control loop is required to determine if this style of feedback results in better control and/or embodiment.

Although the 3D printed backing plate prevented vibrations from being absorbed into the arm cuff, it also appeared to allow vibrations to be transferred from one vibration motor to the other. Improvements may be made by each motor being contained on its own backing plate to reduce transfer of vibrations.

Although testing was conducted for communicating three channels of information to represent a 3-DOA prosthetic hand, we postulate that this system also shows a large potential for 1-DOA and 2-DOA systems, as demonstrated in Table II when the success rate is broken down into individual grip patterns.

The current predominant method of controlling prosthetic hands, is through the use of Electromyography (EMG) signals based off muscles in the forearm. Further testing is therefore required to determine any possible interaction between vibration and the EMG sensors. Since the LRA motors operate based off an AC current and a moving magnet, these may produce electrical signals that could interfere with the EMG signal detection. In addition, the perception of vibration movements may change when the muscle stiffness is altered during retrieving EMG signals.

Further testing will be needed to determine the optimum level of strength and whether user's find 'the always on vibration' useful or too distracting, as reported by a small number of users in some previous studies involving vibration feedback $[22,34]$. A balance may need to be made in whether the stimulations are to be continually sent or only intermittently, in order to minimise the level of distraction. However, it appears this feeling of distraction is not consistent across all users and may also be environment and/or situation dependent. Therefore, these settings may need to be adjusted individually for each patient, and allow each individual user to change their own settings to suit their usage context. Further experimentation is therefore required to determine the 
impact on the accuracy and cognitive load required as a result of intermittent stimulation.

\section{CONCLUSION}

This study demonstrated a new and alternative approach to communicate digit movement information for upper limb prosthetics. Although the prototype of the hardware is large, without the prototype boards, this could easily be built into a small and lightweight embedded system that can be easily attached, removed and position changed on the arm. With a short training period, a mean recognition rate of $72 \%$ (median $78 \%$ ) was achieved with six different grip patterns with two different movement directions. However, with the high grip recognition rate of $84 \%$ and the direction recognition rate of $86 \%$ the system shows potential as a method of sensory feedback.

\section{ACKNOWLEDGMENT}

This research has been conducted with the support of the Australian Government Research Training Program Scholarship, and with the support of the ARC Centre of Excellence for Electromaterials Science (Grant No. CE140100012).

\section{REFERENCES}

[1] R. S. Johansson and J. R. Flanagan, "Coding and use of tactile signals from the fingertips in object manipulation tasks," Nat Rev Neurosci, vol. 10, pp. 345-59, May 2009.

[2] D. J. Atkins, D. C. Y. Heard, and W. H. Donovan, "Epidemiologic Overview of Individuals with Upper-Limb Loss and Their Reported Research Priorities," JPO: Journal of Prosthetics and Orthotics, vol. 8, pp. 2-11, 1996.

[3] E. A. Biddiss and T. T. Chau, "Upper limb prosthesis use and abandonment: A survey of the last 25 years," Prosthetics and Orthotics International, vol. 31, pp. 236-257, 2007.

[4] E. Biddiss and T. Chau, "Upper-limb prosthetics: critical factors in device abandonment," American journal of physical medicine \& rehabilitation, vol. 86, pp. 977-987, 2007.

[5] H. H. Ehrsson, B. Rosen, A. Stockselius, C. Ragno, P. Kohler, and G. Lundborg, "Upper limb amputees can be induced to experience a rubber hand as their own," Brain, vol. 131, pp. 3443-52, Dec 2008.

[6] M. D. Alonzo, F. Clemente, and C. Cipriani, "Vibrotactile Stimulation Promotes Embodiment of an Alien Hand in Amputees With Phantom Sensations," IEEE Transactions on Neural Systems and Rehabilitation Engineering, vol. 23, pp. 450457, 2015.

[7] L. Schmalzl, A. Kalckert, C. Ragno, and H. H. Ehrsson, "Neural correlates of the rubber hand illusion in amputees: a report of two cases," Neurocase, vol. 20, pp. 407-20, Aug 2014.
B. Peerdeman, D. Boere, H. Witteveen, H. Hermens, S. Stramigioli, J. Rietman, et al., "Myoelectric forearm prostheses: State of the art from a user-centered perspective," Journal of Rehabilitation Research and Development, vol. 48, pp. 719-738, 2011.

[9] B. Stephens-Fripp, G. Alici, and R. Mutlu, "A Review of Non-Invasive Sensory Feedback Methods for Transradial Prosthetic Hands," IEEE Access, 2018.

[10] C. J. Hasson and J. Manczurowsky, "Effects of kinematic vibrotactile feedback on learning to control a virtual prosthetic arm," J Neuroeng Rehabil, vol. 12, p. 31, Mar 242015.

[11] M. A. Schweisfurth, M. Markovic, S. Dosen, F. Teich, B. Graimann, and D. Farina, "Electrotactile EMG feedback improves the control of prosthesis grasping force," J Neural Eng, vol. 13, p. 056010, Oct 2016.

[12] A. Ninu, S. Dosen, S. Muceli, F. Rattay, H. Dietl, and D. Farina, "Closed-Loop Control of Grasping With a Myoelectric Hand Prosthesis: Which Are the Relevant Feedback Variables for Force Control?," IEEE Transactions on Neural Systems and Rehabilitation Engineering, vol. 22, pp. 10411052, 2014.

[13] H. J. Witteveen, E. A. Droog, J. S. Rietman, and P. H. Veltink, "Vibro- and electrotactile user feedback on hand opening for myoelectric forearm prostheses," IEEE Trans Biomed Eng, vol. 59, pp. 2219-26, Aug 2012.

H. Yamada, Y. Yamanoi, K. Wakita, and R. Kato, "Investigation of a cognitive strain on hand grasping induced by sensory feedback for myoelectric hand," in 2016 IEEE International Conference on Robotics and Automation (ICRA), 2016, pp. 3549-3554.

M. Nabeel, "Vibrotactile stimulation for 3D printed prosthetic hand," in Robotics and Artificial Intelligence (ICRAI), 2016 2nd International Conference on., 2016, pp. 202-207.

[16] T. Rosenbaum-Chou, W. Daly, R. Austin, P. Chaubey, and D. A. Boone, "Development and Real World Use of a Vibratory Haptic Feedback System for Upper-Limb Prosthetic Users," JPO: Journal of Prosthetics and Orthotics, vol. 28, pp. 136-144, 2016.

[17] P. Chaubey, T. Rosenbaum-Chou, W. Daly, and D. Boone, "Closed-Loop Vibratory Haptic Feedback in Upper-Limb Prosthetic Users," JPO: Journal of Prosthetics and Orthotics, vol. 26, pp. 120-127, 2014.

[18] T. Li, H. Huang, C. Antfolk, J. Justiz, and V. M. Koch, "Tactile display on the remaining hand for unilateral hand amputees," Current Directions in Biomedical Engineering, vol. 2, 2016.

E. Raveh, J. Friedman, and S. Portnoy, "Visuomotor behaviors and performance in a dualtask paradigm with and without vibrotactile 
feedback when using a myoelectric controlled hand," Assist Technol, pp. 1-7, Jun 192017.

[20] H. J. Witteveen, H. S. Rietman, and P. H. Veltink, "Vibrotactile grasping force and hand aperture feedback for myoelectric forearm prosthesis users," Prosthet Orthot Int, vol. 39, pp. 204-12, Jun 2015.

[21] A. Ajoudani, S. B. Godfrey, M. Bianchi, M. G. Catalano, G. Grioli, N. Tsagarakis, et al., "Exploring teleimpedance and tactile feedback for intuitive control of the Pisa/IIT SoftHand," IEEE Trans Haptics, vol. 7, pp. 203-15, Apr-Jun 2014.

[22] M. C. Jimenez and J. A. Fishel, "Evaluation of force, vibration and thermal tactile feedback in prosthetic limbs," in 2014 IEEE Haptics Symposium (HAPTICS), 2014, pp. 437-441.

[23] P. D. A.-O. h. o. o. Marasco, J. S. A.-O. h. o. o. Hebert, J. W. A.-O. h. o. o. Sensinger, C. E. Shell, J. S. A.-O. h. o. o. Schofield, Z. C. A.-O. h. o. o. Thumser, et al., "Illusory movement perception improves motor control for prosthetic hands.," Science Translational Medicine, 2018.

[24] K. Bark, J. Wheeler, P. Shull, J. Savall, and M. Cutkosky, "Rotational Skin Stretch Feedback: A Wearable Haptic Display for Motion," IEEE Trans Haptics, vol. 3, pp. 166-176, Jul-Sep 2010.

[25] E. Battaglia, J. P. Clark, M. Bianchi, M. G. Catalano, A. Bicchi, and M. K. O'Malley, "The Rice Haptic Rocker: skin stretch haptic feedback with the Pisa/IIT SoftHand," in World Haptics Conference (WHC), 2017 IEEE, 2017, pp. 7-12.

[26] A. Akhtar, M. Nguyen, L. Wan, B. Boyce, P. Slade, and T. Bretl, "Passive mechanical skin stretch for multiple degree-of-freedom proprioception in a hand prosthesis," in International Conference on Human Haptic Sensing and Touch Enabled Computer Applications, 2014, pp. 120-128.

[27] S. Yao and Y. Zhu, "Nanomaterial-Enabled Dry Electrodes for Electrophysiological Sensing: A Review," JOM, vol. 68, pp. 1145-1155, April 01 2016.

[28] H. E. Burtt, "Tactual illusions of movement," Journal of Experimental Psychology, vol. 2, pp. 371-385, 1917.

[29] J. H. Kirman, "Tactile apparent movement: The effects of interstimulus onset interval and stimulus duration," Perception \& Psychophysics, vol. 15, pp. 1-6, January 011974.

[30] A. Israr and I. Poupyrev, "Control space of apparent haptic motion," presented at the 2011 IEEE World Haptics Conference, 2011.

[31] J. H. Kirman, "Tactile apparent movement: the effects of number of stimulators," J Exp Psychol, vol. 103, pp. 1175-80, Dec 1974.

[32] J. Cohen, M. Niwa, R. W. Lindeman, H. Noma, Y. Yanagida, and K. Hosaka, "A closed-loop tactor frequency control system for vibrotactile feedback," in CHI'05 Extended Abstracts on Human Factors in Computing Systems, 2005, pp. 1296-1299.
[33] T. A. Kuiken, G. Li, B. A. Lock, R. D. Lipschutz, L. A. Miller, K. A. Stubblefield, et al., "Targeted Muscle Reinnervation for Real-Time Myoelectric Control of Multifunction Artificial Arms," JAMA : the journal of the American Medical Association, vol. 301, pp. 619-628, 2009.

[34] Z. Ying and J. B. Morrell, "A vibrotactile feedback approach to posture guidance," in 2010 IEEE Haptics Symposium, 2010, pp. 351-358. 